\title{
The influence of alcohol and loud music on analytic and holistic processing
}

\author{
THOMAS B. WARD and SHERRI N. LEWIS \\ Texas A\&M University, College Station, Texas
}

\begin{abstract}
The influence of alcohol and loud music on analytic versus holistic processing was examined in classification, concept learning, and embedded figures tasks. In the classification task, loud music reduced analytic responding by individuals who had consumed alcohol and increased analytic responding by individuals who had received placebo drinks. Alcohol also decreased the speed with which individuals were able to generate analytic responses in the classification task and decreased analytic performance on the embedded figures test. Alcohol slowed the rate of learning in the concept-learning task but did not lead to less analytic responding in that task. The results are consistent with the idea that alcohol slows the rate of information processing, particularly in tasks requiring visual analysis of stimuli.
\end{abstract}

The ability to effectively allocate attention has been widely cited as being crucial to safe driving (e.g., Kahneman, Ben-Ishai, \& Lotan, 1973; Mihal \& Barrett, 1976; Mitchell, 1985; Shinar, 1978). For example, in operating a motor vehicle, the driver must attend to the visual cues that are relevant for keeping the car on the road at a safe distance from other cars and avoid being distracted by irrelevant visual and auditory information. Because of the importance of selective attention in driving and in performing other potentially dangerous tasks, any factor that interferes with the ability to selectively process relevant information increases the probability of serious injury.

Because of the evident link between alcohol consumption and automobile accidents (see, e.g., U.S. Department of Transportation, 1985), as well as for theoretical reasons, a considerable amount of research has been conducted to document the negative effects of alcohol on attention. Despite this research effort there are still major gaps in our knowledge. Particularly lacking are studies concerned with the interactive effects of alcohol in combination with common distractors, such as loud music, that are likely to be present in many real-world settings. The present research is an attempt to fill some of those gaps by examining both the separate and combined effects of alcohol and loud music on the individual's ability or tendency to selectively process relevant information. The present research also introduces an alternate way of conceptualizing the impact of alcohol on performance in tasks demanding selective attention.

This paper is based on a senior thesis conducted by the second author under the direction of the first author. The authors thank Claude Goswick and the A. P. Buetel Health Center staff for their willing cooperation and for the accommodations they provided for the subjects, and Nick Pace for the use of his laboratory, equipment, and supplies. We also thank Marshall Divita, Jana Rmeili, Laura Roush, and other students without whose technical assistance this study would not have been possible. Address correspondence to: Thomas B. Ward, Department of Psychology, Texas A\&M University, College Station, TX 77843.
Our reasons for including music as a distractor are largely practical, but there is also empirical and theoretical justification for doing so. From a practical perspective, it is reasonable to suppose that many individuals who drive under the influence of alcohol also listen to music while doing so. Therefore, it is crucial to determine whether or not the effects of alcohol on attentional performance are even greater in the presence of such a potential distractor. From an empirical perspective, there is reason to predict just such an effect. For example, Hamilton and Copeman (1970) demonstrated that individuals under the influence of alcohol were adversely affected by extraneous noise. Although music is more structured than "noise," it seemed reasonable to predict on the basis of such findings that individuals who consumed alcohol in the present study would show greater declines in performance due to exposure to loud music than would individuals who received placebo drinks.

Although there is some disagreement in the literature (see, e.g., MacArthur \& Sekuler, 1982), much research is consistent with the idea that alcohol has a negative effect on the individual's ability to divide attention between two or more relevant sources of information (Moskowitz, 1973; Moskowitz \& DePry, 1968; Moskowitz \& Sharma, 1974; Shinar, 1978). In addition, there is suggestive evidence that alcohol may interfere with the ability to selectively allocate attention to one relevant source of information and ignore other, irrelevant sources (see, e.g., Hamilton \& Copeman, 1970; Harley, Cohen, \& Silverman, 1974; Kristofferson, 1968).

The studies on both divided and selective attention are certainly consistent with the idea that alcohol has a negative effect on the process of attention itself. The present research, however, offers an alternative way of conceptualizing the negative effects of alcohol in attention tasks, particularly those requiring selective attention. It is possible, as elaborated more fully below, that alcohol induces individuals to adopt a more holistic mode of perceiving, 
which in turn limits their ability to attend selectively to some aspects of stimulation and to ignore others.

The major theoretical underpinning of the present study is the work of Garner $(1970,1974$; see also Lockhead, 1966; Shepard, 1964) and subsequent modifications of that work (Shepp, 1978; J. D. Smith \& Kemler Nelson, 1984; L. B. Smith \& Kemler, 1977; Ward, 1983, 1985; Ward, Foley, \& Cole, 1986). Gamer pointed out that models of information processing and, in particular, models of selective attention could not be specified without reference to the type of stimulus dimensions being processed. Garner and others have proposed that when stimuli are composed of separable dimensions (e.g., the size and brightness of geometric forms), those dimensions are perceived as distinct entities and individuals are both able and likely to process the dimensions independently of one another. Thus, separable perception can be viewed as analytic. Integral dimensions (e.g., hue and saturation), in contrast, are perceived as unitary. Individuals have a great deal of difficulty in responding to the component dimensions of a stimulus when those dimensions are integral, and they are more likely to respond to such a stimulus in a unitary way. Therefore, integral perception can be thought of as holistic. A crucial aspect of the separableintegral distinction for the present research is that individuals are readily able to attend selectively to the component features or dimensions of separable materials but not to integral materials.

Although the separable-integral distinction originally described different types of stimuli, it has recently been used successfully to describe differences in human performance under various task conditions. The bulk of that research indicates that a holistic, integral mode of processing is a more primitive, less developed one that is often, if not always, associated with poor selective-attention performance. For example, when adults are required to perform subsidiary tasks, their performance in classification tasks becomes more holistic (J. D. Smith \& Kemler Nelson, 1984). Presumably this is because performing the subsidiary task limits the resources they can apply to the main task. In addition, even without subsidiary tasks, there are clearly some adults who take a holistic, integral approach to tasks, and, presumably as a result of that approach, those individuals perform more poorly in selective-attention tasks than do individuals who adopt a more analytic, separable mode of processing (Ward, 1985; Ward et al., 1986).

The separable-integral approach has also been used effectively in studies of individuals of different ages and abilities. Young children, for example, are more likely than older children and adults to adopt an integral approach to tasks (Shepp, 1978; L. B. Smith \& Kemler, 1977; Ward, 1980). In addition, learning-disabled (Ward, 1986b), hyperactive (Fisher, 1977), and retarded (Kemler, 1982) individuals appear to perceive stimuli in a more integral manner. Thus, the poor performance typically shown by those groups in tasks of selective attention may be related as much to a more holistic mode of perception as it is to a deficit in the attentional process per se. By analogy, the effects of alcohol on performance in tasks that require selective attention may be the result of alcohol's shifting individuals to the more primitive holistic, integral mode of perceiving. Since alcohol has a wide range of negative effects on cognitive and perceptual functioning (see, e.g., Mitchell, 1985; U.S. Department of Transportation, 1985), it is reasonable to predict that alcohol will lead individuals to be unable to apply the processing resources needed for the more advanced analytic, separable mode.

An alternative way of conceptualizing the effect of alcohol is that individuals who have consumed alcohol will be able to be analytic but will require more time to analyze incoming information. That is, even in sober adults, there is evidence that in the processing of any given stimulus, accessing analytic information about specific component dimensions or features of the stimulus requires extended processing time (J. D. Smith \& Kemler Nelson, 1984; Ward, 1983; Ward et al., 1986). Since alcohol has been shown to reduce rate of information processing (see, e.g., Moskowitz \& Burns, 1971; Moskowitz \& Murray, 1976), it is possible that adults under the influence of alcohol will show just as much analytic, separable responding as sober adults but take more time in doing so. Such an effect would be just as likely to lead to poor driving performance as the effect described in the previous paragraph, since, under driving conditions, an individual would presumably have a limited amount of time to decompose holistic inputs into their relevant components.

A study was conducted to assess the impact of alcohol on analytic, separable and holistic, integral modes of processing. Three tasks were used. The first was the restricted classification task, in which participants are shown triads of stimuli on each trial. Their task is to indicate the two out of three items that go together best. Triads are constructed from multidimensional stimuli so that classifications made on the basis of similarity can be differentiated from those made on the basis of identity along one of the component dimensions. Similarity classifications are taken as an indication of integral perception and dimensional classifications are taken as an indication of separable perception (Garner, 1974; L. B. Smith \& Kemler, 1977; Ward, 1980, 1983). To examine the issue of whether people under the influence of alcohol are unable to be analytic or simply require more time to be so, our participants were timed as they performed the classification task. The second task was the concept-learning task described by Kemler Nelson (1984). In that task, the individual can learn the concept either by an analytic singleattribute rule or by a holistic, overall-appearance rule. Of interest was whether or not alcohol would induce adults to adopt the latter type of rule. In the final task, the Group Embedded Figures Test (GEFT; see Oltman, Raskin, \& Witkin, 1971) was used as a measure of ability to separate the component features of a stimulus. To the extent that alcohol has the very general effect of shifting people toward more holistic modes of processing, we should ex- 
pect the performance of people who have consumed alcohol to be less analytic across all three tasks. In addition, to the extent that alcohol reduces the ability to resist distraction, we should expect even less analytic performance in individuals who are also exposed to the distraction of music while performing those tasks.

To summarize, the present research sought, in several ways, to add to our knowledge of the effects of alcohol on attention. First, it was concerned with alcohol's effect on a person's ability or tendency to selectively process some of the component features or dimensions of presented stimuli but not others. Second, it investigated the possibility that the effects of alcohol on performance in tasks of attention are mediated by a shift to a more holistic mode of perception. Third, it included a distractor (i.e., loud music) that could reasonably be assumed often to be present when people drink and drive and that could interact with alcohol to produce even stronger effects than alcohol alone. Such interactive effects are rarely considered in research on the effects of alcohol.

\section{METHOD}

\section{Participants}

Fifty-two undergraduate males enrolled in introductory psychology classes at Texas A\&M University participated for course credit. By self-report, the participants were social drinkers who had never become violent while drinking and had no medical condition that would preclude the use of alcohol.

\section{Stimuli}

Restricted classification tasks. Three sets of multidimensional stimuli were prepared for use in the restricted classification task: circles that varied in area and radius orientation, lines of dots that varied in length and density, and squares that varied in size and brightness. Although these stimulus materials may seem to be extremely simple, it should be noted that they are the types of items that are used most extensively in studies of separable and integral perception. In addition, they have proven to be useful in clarifying the nature of poor attentional performance in young children (Shepp, 1978; Shepp, Burns, \& McDonough, 1980; L. B. Smith \& Kemler, 1977; Ward, 1980), in individuals with certain disabilities (Fisher, 1977; Kemler, 1982; Ward, 1986b), and in certain groups of field-dependent adults (Ward, 1985; Ward et al., 1986). Thus, there was good reason to believe that these materials would be useful in clarifying the nature of alcohol-induced attention deficits as well.

For each of the three sets, triads were constructed that would allow classifications based on dimensional identity to be differentiated from those based on overall similarity. The structure of the triads can be illustrated by the example of three possible pairings of items given in Figure 1. Two items in each triad were identical on one dimension and very different on the other ( $A$ and $B$ in Figure 1). These items are referred to as the dimensional pair. The third item in each triad ( $C$ in Figure 1 ) differed from each of the others on both dimensions. The third item was highly similar to one of the other items (similarity pair, $A$ and $C$ ) and very different from the other one (haphazard pair, B and C). Similarity rating data reported by Burns, Shepp, McDonough, and Wiener-Ehrlich (1978) and Ward (1978) confirm that the similarity pair is perceived as more similar than either the dimension pair or the haphazard pair for each triad.

The circles were $4.5,6.4,9.0,12.8,18.8$, or $25.8 \mathrm{sq} \mathrm{cm}$ in area and had radius orientations of $5^{\circ}, 35^{\circ}, 65^{\circ}, 95^{\circ}, 125^{\circ}$, or $155^{\circ}$ from vertical. The circles and their radii were computer-drawn and

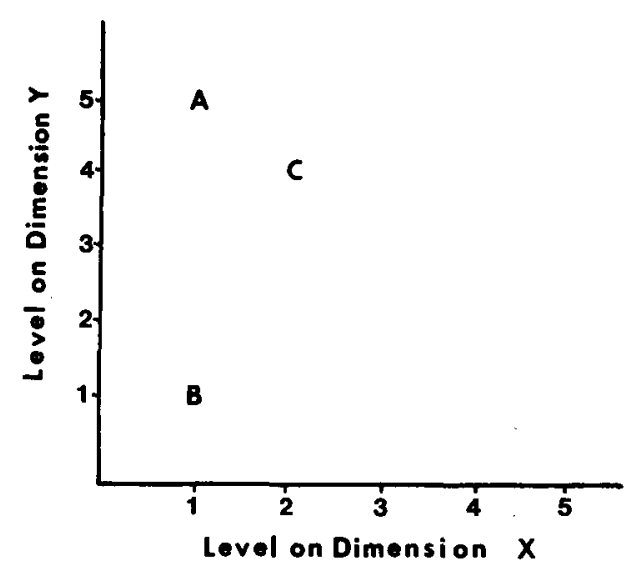

Figure 1. Representation of a triad of stimuli for the restricted classification task.

printed in black on white backgrounds. The radii always extended $8 \mathrm{~mm}$ in length from the center of the circles regardless of their area. Each triad of circles was arranged in a single horizontal row across the long axis of an $8.5 \times 11$ in. white sheet of paper. Eight distinct triads were constructed, with four having area as the dimensional response and four having radius orientation as the dimensional response. Three versions of each triad were constructed so that each triad member could appear in each position on the sheet and each response type (dimensional, similarity, and haphazard) could appear in each possible pairing of positions on the sheet. This resulted in a total of 24 circle-triad presentations.

The squares, which varied in size and brightness, were cut from Munsell glossy standards. Triads were chosen from the possible combinations of side lengths of 2, 2.5, 3, 4, and $5 \mathrm{~cm}$ and Munsell neutral gray values N4-N7. The squares of each triad were attached in a horizontal row on an $8.5 \times 11$ in. sheet of white paper. Eight triads were constructed, with four having side length as the dimensional response and four having brightness as the dimensional response. Three versions of each square triad were constructed, as described for the circle triads.

The third set of materials consisted of lines of dots that varied in line length and density of the dots. The lines were $1,1.5,2$, $3,4,6$, or $8 \mathrm{~cm}$ in length and had densities of $1.5,1, .75, .50$, .25 , or $.125 \mathrm{~cm}$ interdot distance. The lines of a given triad were typed one above the other and centered on a $3 \times 5$ in. white index card. Eight triads were constructed, with four having length as the dimensional response and four having density as the dimensional response. As with the circles and squares, three versions of each triad were constructed.

Concept learning. The materials and task used for concept learning were patterned after those used by Kemler Nelson (1984, Experiment 2). Cartoon faces that varied in the attributes of hair, ears, nose, and mustache were created. The different levels of each attribute were designated by the codes 1,2 , and 3 , and the coding scheme was as depicted in Table 1. An example of the concept problems used is given in Table 2. Note that each category contains three exemplars and that the members of category $A$ all have straight hair, whereas the members of category B all have curly hair. Furthermore, the characteristics in category $A$ tend toward one extreme level of attributes and the characteristics of category B tend toward the other. According to Kemler Nelson's (1984) analysis, the problem can be approached in one of two ways: With an analytic approach, the attribute of hair alone can be used, with a 1 or a 3 cuing a category A or category B classification, respectively. With a holistic approach, the choice can be made on the basis of the number of coded characteristics that lie at one or the other 
of the extremes, with the presence of two or more 1 s deciding the issue for category $A$ and two or more $3 \mathrm{~s}$ deciding the issue for category B [that is, in overall appearance, the faces in category $A$ (B) are more similar to each other in terms of the characteristics set forth in Table 1 than they are to the faces in category $B(A)]$. To test which approach the person has adopted, critical test faces of the form 1333 and 3111 are used. If the person has focused on the single attribute of hair, then he should call $1333 \mathrm{~A}$ and 3111 $B$. If the person has focused on overall appearance, he should call $3111 \mathrm{~A}$ and $1333 \mathrm{~B}$. The full set of test items used for the problem shown in Table 2 is given in Table 3. Note that PA and PB are the prototypic faces for categories $\mathbf{A}$ and $\mathbf{B}$, respectively; that is, they have the characteristic level for all four attributes. T1 and T2 are the critical test items, and the remaining faces are closely related to those test faces.

Sixteen concept problems were created in the following manner. Four pairs of prototype faces were created such that, across all eight of those faces, each extreme level ( 1 or 3 ) of each attribute was paired equally often with each extreme level of the other three attributes. For each of those prototype pairs, four separate problems were created such that each attribute could serve equally often as the potentially critical attribute.

Other materials. The third phase of the study involved the administration of the GEFT (Oltman al., 1971). The standard GEFT booklets that were used contain one page depicting simple geometric figures and several pages depicting complex geometric figures. Each complex figure has one of the simple figures "hidden" within it, and the participant's task is to locate the simple figure within each complex figure. The booklets are divided into a practice section and two test sections, each containing nine complex geometric figures.

In addition, tapes of music, recorded with the assistance of a local radio station, were used. These included selections of either the Top 40 rock songs or contemporary country songs.

\section{Procedure}

Prior to the study, each potential participant was given a confidential questionnaire concerning prior drug and alcohol use. Subjects who reported consuming alcohol less than one or two times per week or more than four times per week and ones who reported consuming more than four drinks in one sitting were not accepted. Prior to the experiment, all subjects were required to take a physical exam. In addition, the subjects were asked to use no drugs, except caffeine and cigarettes, $\mathbf{4 8}$ hours prior to the experiment; they were asked to limit their use of caffeine and cigarettes as much as possible during this time. They were also asked to consume only clear liquids and no solid foods during the $8 \mathrm{~h}$ preceding the experiment.

Table 1

Coding Scheme Used for Faces in Concept-Learning Task

\begin{tabular}{cllll}
\hline & \multicolumn{4}{c}{ Attribute/Type } \\
\cline { 2 - 5 } Level & Hair (H) & Mustache (M) & Ears (E) & Nose (N) \\
\hline 1 & straight & clipped & small & tall-thin \\
2 & wavy & medium-length & medium & medium \\
3 & curly & handlebar & large & short-fat \\
\hline
\end{tabular}

Table 2

Representation of Learning Items Used for Concept-Learning Task

\begin{tabular}{cccccccc}
\hline & \multicolumn{3}{c}{ Category A } & & \multicolumn{4}{c}{ Category B } \\
\cline { 6 - 8 } H & M & E & N & H & M & E & N \\
\hline 1 & 1 & 1 & 2 & 3 & 3 & 3 & 2 \\
1 & 1 & 2 & 1 & 3 & 3 & 2 & 3 \\
1 & 2 & 1 & 1 & 3 & 2 & 3 & 3 \\
\hline
\end{tabular}

Note-The attributes are hair $(H)$, mustache $(M)$, ears $(E)$, and nose $(N)$.
Table 3

Transfer Items for Concept-Learning Task

\begin{tabular}{ccccc}
\hline & \multicolumn{4}{c}{ Item } \\
\cline { 2 - 5 } Label & H & M & E & N \\
\hline PA & 1 & 1 & 1 & 1 \\
PB & 3 & 3 & 3 & 3 \\
T1 & 3 & 1 & 1 & 1 \\
T2 & 1 & 3 & 3 & 3 \\
T3 & 3 & 1 & 1 & 2 \\
T4 & 1 & 3 & 3 & 2 \\
T5 & 3 & 1 & 2 & 1 \\
T6 & 1 & 3 & 2 & 1 \\
T7 & 3 & 2 & 1 & 1 \\
T8 & 1 & 2 & 3 & 3 \\
\hline
\end{tabular}

Note-The attributes are hair $(H)$, mustache $(M)$, ears $(E)$, and nose (N). PA and PB are category A and category B prototypes, respectively. T1-T8 are the critical test faces.

Since the experiment involved the consumption of alcohol, it was conducted at the campus health center, and the participants given alcohol were required to remain at the health center overnight as inpatients. They were released by a physician the next morning. Since they had been asked to consume no solid foods during the previous $8 \mathrm{~h}$, all participants were provided with supper following the experiment. The participants were interviewed upon their arrival at the health center to determine what drugs (including prescription and nonprescription medications and alcohol) they might have taken in the previous $48 \mathrm{~h}$ and what food they had eaten in the previous $12 \mathrm{~h}$. Potential participants who failed to meet the established requirements were rescheduled for participation at a later date.

After reading and signing informed consent forms, the participants were given one Carnation instant breakfast (vanilla) mixed in $8 \mathrm{oz}$ of whole milk. The participants were then asked to wait and were given the choice of one of the types of music to listen to while they waited. For participants in the music conditions, the type of music chosen at this point was the type used as a distractor in the experiment proper. ${ }^{1}$ One hour after consuming the instant breakfast, the subjects were given their first drink. They were given one additional drink every $15 \mathrm{~min}$ for $1 \mathrm{~h}$ (i.e., a total of five drinks). Twenty minutes later, the subjects were given their sixth and final drink. Participants began performing the tasks after the fifth drink.

To make the drinks, 1 liter of lemonade was mixed using $150 \%$ of the recommended amount of Minute Maid lemonade crystals. To this, $1.25 \mathrm{ml}$ of peppermint extract was added to create a masking solution. Alcoholic drinks contained $.20 \mathrm{ml}$ of $95 \%$ grain alcohol per kilogram of body weight combined with five parts of masking solution. Placebo drinks contained the same volume of liquid as the alcohol-containing drinks but consisted of $100 \%$ masking solution. The rim of each cup containing a placebo drink was swabbed with a small amount of alcohol.

Before the administration of any placebo or alcohol-containing drinks, blood was drawn for later analysis to confirm that the blood alcohol concentration (BAC) was zero. Additional BAC samples were taken just prior to the restricted classification task, just after completion of the concept-learning task, and immediately following completion of the GEFT.

The blood samples were taken by an emergency medical technician trained in that procedure, and an emergency-care attendant was present at all times to monitor subjects and handle any emergencies that might occur. Venipuncture sites were swabbed with Betadine solution to avoid interference of skin-surface alcohol with blood alcohol determination. Blood was collected in 7.5 -cc vacutainer tubes that contained EDTA anticoagulant and were centrifuged to obtain plasma samples. Blood alcohol concentrations were determined according to Procedure 332-UV of Sigma Diagnostics. 
The study involved a 2 (alcohol vs. placebo) $\times 2$ (loud music vs. no music) design. Thirteen subjects were tested in each of the four cells. Although participants in the music conditions were given a choice of what type of music they wanted to listen to, nearly all chose the Top $\mathbf{4 0}$ rock songs. In the music conditions, the experimenter adjusted the volume of the tape so that it was loud enough to be potentially distracting without being so loud that the instructions and the participants' responses were unintelligible. The participants were not told to try to ignore or attend to the music.

The experiment was double-blind with neither the tester nor the subject knowing whether a placebo or an alcohol-containing drink was being administered. The project supervisor was the only person who knew which subjects received placebo drinks and which received alcohol-containing drinks.

The participants were tested individually in a small room in the health center. All performed a restricted classification task with all three sets of materials, then performed the concept-learning task with one of the 16 possible problem types, and then completed the GEFT. The fixed order of task presentation was used because previous research had indicated that the classification task is sensitive to practice effects and to a number of other factors that affect the participant's approach to the task (see, e.g., J. D. Smith \& Kemler Nelson, 1984; Ward, 1983, 1985). In contrast, the conceptlearning task appears much less sensitive to such factors, including explicit instructions to adopt particular strategies (see, e.g., Kemler Nelson, 1984; Medin \& E. E. Smith, 1981), and the GEFT, which has a strong ability component (see, e.g., Kogan, 1973), should be least influenced. We hoped to avoid a potential analytic bias on the classification task, which might result if the participants performed the other tasks first.

Within the restricted classification task, the participants classified all triads for one set of materials before classifying another set. Order of presentation of the sets of materials was counterbalanced across participants such that, as nearly as possible, each of the three types of material occurred equally often in the first, second, and third presentation positions.

In the restricted classification task, participants were asked to pick, for each triad, the two out of three items that "go together best" or "belong in the same group." The left, middle, and right (or top, middle, and bottom) positions in the triad were assigned the labels $a, b$, and c, respectively, and participants indicated their choices by pointing and by verbalizing the appropriate pair of letters.

For all types of materials, the triads were presented in three trial blocks. That is, the 8 distinct triads were all presented in a random order (Trial Block 1), then in another random order (Trial Block 2), and then again in a third random order (Trial Block 3). There was no hesitation between trial blocks, so the participants received a continuous sequence of 24 presentations for each set of materials. The experimenter used a stopwatch to time participants' 24 judgments for each of the three sets of materials.

In the learning phase of the concept-learning task, the participants were told that there was an imaginary world in which firemen and policemen could be distinguished on the basis of appearance, and that it was their task to learn which faces were firemen and which were policemen. The participants were given 36 learning trials, on each of which they were presented with one of the six learning faces and asked to indicate whether the face was that of a fireman or policeman. They were given feedback in each case. The 36 learning trials were structured so that each learning face occurred once in each block of six trials. In the transfer phase, the participants were to continue indicating whether faces were those of firemen or policemen, but feedback was discontinued. A total of 32 transfer trials were presented. Included were two presentations each of the 6 original learning faces, 2 prototypes, 2 critical test faces, and 6 close relations of the test faces. Each face occurred once in each of two blocks of 16 transfer trials.
After completing the restricted classification task, with all three sets of material, and the concept-learning task, the participants were given the GEFT according to standard procedures (Oltman et al., 1971). After completing the practice section of the GEFT, the participants were allowed $5 \mathrm{~min}$ for each of the test sections.

\section{RESULTS}

\section{Blood Alcohol Concentrations}

Analysis of the blood alcohol data indicated that the mean BAC level was $.048 \%$ just prior to the classification task, $.073 \%$ just after the concept-learning task, and $0.84 \%$ just after the GEFT. Thus, although BACs were higher for the GEFT and concept-learning tasks, all tasks occurred on the ascending limb of the BAC curve.

\section{Restricted Classification}

The mean number of similarity (holistic), dimensional (analytic), and haphazard responses averaged across the three types of materials in the restricted classification task is presented in Table 4 separately for each of the four groups. Since there was no interaction between the three types of materials and either the alcohol or music variable, the results are not broken down for each of the materials separately. An analysis of variance conducted on the number of holistic responses revealed that the only significant effect was the alcohol $\times$ music interaction $[F(1,48)=4.90, p<.05]$. Although none of the individual mean comparisons achieved statistical significance, as shown in Table 4, the pattern of the interaction is that music reduced the amount of holistic responding by subjects who received placebo drinks but increased holistic responding by those who received alcohol. An analysis on the analytic, dimensional responses also revealed a significant alcohol $\times$ music interaction $[F(1,48)=4.13, p<.05]$. Inspection of Table 4 reveals that music increased analytic responding by the placebo group and decreased analytic responding by the alcohol group.

Careful inspection of Table 4 reveals that the alcohol group was, on the average (i.e., collapsing across the music conditions), neither more nor less holistic than the placebo group in the classification task. However, it is also clear that the alcohol group took much longer to

\section{Table 4}

Performance of the Four Groups on Varions Aspects of the Restricted Classification Tasks

\begin{tabular}{lcccc}
\hline & \multicolumn{4}{c}{ Group } \\
\cline { 2 - 5 } Response Type & $\begin{array}{c}\text { No Alcohol } \\
\text { No Music }\end{array}$ & $\begin{array}{c}\text { No Alcohol } \\
\text { Music }\end{array}$ & $\begin{array}{c}\text { Alcohol } \\
\text { No Music }\end{array}$ & $\begin{array}{c}\text { Alcohol } \\
\text { Music }\end{array}$ \\
\hline Holistic & 7.2 & 5.0 & 5.0 & 7.4 \\
Analytic & 14.6 & 17.3 & 17.2 & 14.3 \\
Haphazard & 2.2 & 1.6 & 1.18 & 2.6 \\
Time (sec) & 138 & 156 & 209 & 198 \\
Analytic/sec & 0.13 & 0.13 & 0.11 & 0.08 \\
\hline
\end{tabular}

Note-The entries for Holistic, Analytic, and Haphazard are the mean numbers of each of those types of responses out of a maximum possible of 24 . 
achieve the same level of analytic performance $[F(1,48)$ $=10.85, p<.01]$. The last row in Table 4 , which presents the number of analytic responses per unit of time, is the most revealing. An analysis of variance conducted on the number of dimensional responses per unit of time revealed a significant effect of alcohol condition $[F(1,48)$ $=7.05, p<.05$ ]. As shown in Table 4, the placebo groups were the most efficient in quickly analyzing the materials into their component dimensions. Although there was no alcohol $\times$ music interaction, the means shown in Table 4 suggests that the alcohol-music group performed worst of all on this measure.

\section{Concept Learning}

Several analyses were performed on the data from the concept-learning task. Speed of learning was assessed by determining the first block of six learning trials in which the participant gave six consecutive correct responses. An analysis of variance revealed that there were marginally significant effects of both alcohol $[F(1,48)=3.27$, $p=.077]$ and music $[F(1,48)=3.27, p=.077]$. Table 5 shows that both alcohol and music resulted in slightly more trial blocks to the criterion of six-in-a-row correct. Table 5 also indicates the number of responses to the critical test faces and the close relations that were consistent with an analytic approach. As indicated by those means, music had a significant effect on the tendency to make analytic choices for the critical test faces $[F(1,48)=4.15$, $p<.05]$ but alcohol did not $(F<1)$. Neither alcohol nor music affected responding to the close-relation faces $[F(1,48)=1.02, p>.30$, and $F(1,48)=2.66, p>.10$, respectively]. The alcohol groups did not take significantly longer $(F<1)$ and did not show fewer analytic choices per unit of learning time $(F<1)$. On the sole basis of responses to the critical test items, 16 of the placebo subjects showed purely analytic patterns and 7 showed holistic patterns. The respective numbers for the alcohol group were 12 and 8 . Thus, there is a slight trend toward less analytic responding in the alcohol group, but the trend is not significant.

\section{Group Embedded Figures Test}

An analysis of variance on the GEFT scores revealed a significant effect only of alcohol $[F(1,48)=8.72$, $p<.01$ ], with the placebo group (mean $=13.9$ out of a possible 18) outperforming the alcohol group (mean =

Table 5

Performance of the Four Groups on Various Aspects of the Concept-Learning Task

\begin{tabular}{lcccc}
\hline & \multicolumn{4}{c}{ Group } \\
\cline { 2 - 5 } \multicolumn{1}{c}{ Measure } & $\begin{array}{c}\text { No Alcohol } \\
\text { No Music }\end{array}$ & $\begin{array}{c}\text { No Alcohol } \\
\text { Music }\end{array}$ & $\begin{array}{c}\text { Alcohol } \\
\text { No Music }\end{array}$ & $\begin{array}{c}\text { Alcohol } \\
\text { Music }\end{array}$ \\
\hline Trial Block & 1.85 & 2.38 & 2.43 & 3.15 \\
Critical Test & 3.08 & 2.46 & 2.79 & 1.77 \\
Close Relation & 9.38 & 6.69 & 8.36 & 5.38 \\
\hline
\end{tabular}

Note-Entries for critical test and close relation indicate the number of responses on transfer trials consistent with an analytic approach. The maximum possible for the former is 4 and for the latter, 12.
9.9). The result is consistent with those of previous research showing a relationship between alcohol and field dependence (e.g., Harley et al., 1974; Kristofferson, 1968) and with the idea that people under the influence of alcohol are less able to separate incoming stimulation into relevant and irrelevant components.

\section{DISCUSSION}

The results of the present study reveal striking effects of alcohol on performance in two of the three tasks presented. They also reveal a potentially important interaction of alcohol and music on classification performance.

Although alcohol did not reduce the number of analytic classifications that participants made in the restricted classification task, it did increase response time and lead to fewer analytic responses per unit of processing time. Since alcohol tends to have little effect on simple reaction time, the effect observed can be viewed as being based on a slowing of the perceptual processes required for task performance (see, e.g., Mitchell, 1985). Apparently, people under the influence of alcohol are capable of analyzing stimuli into components, but they require an unusually long period of time to do so. Although the trend did not achieve statistical significance, the mean differences observed provide a hint that the presence of music as a distractor may make the situation even worse. Since driving, especially at high speeds, may require rapid analysis of incoming stimuli, this effect could be related to an increase in number of accidents.

On the surface, there appears to be a discrepancy between the results for the classification task and those for the GEFT, in that the latter revealed an overall performance deficit, whereas the former revealed an effect only when processing time was considered. However, it is important to note that, in contrast to the classification task, which allows the participant to determine rate of performance, the GEFT has a fixed time limit (10 min). Thus, the overall effect of alcohol on GEFT performance may be consistent with the classification results, which suggested a slower rate of analysis. Such an effect is consistent with the results of previous studies (e.g., Moskowitz \& Burns, 1971; Moskowitz \& Murray, 1976) that have revealed that alcohol slows the rate of information processing.

The results of the present study also mimic findings from studies of individual and developmental differences. For example, Ward et al. (1986) found that sober individuals who performed poorly on the GEFT also had slower rates in extracting dimensional information in a restricted classification task under standard conditions with no distraction. Likewise, elderly individuals, who perform poorly on embedded figures tests (see, e.g., Axelrod \& Cohen, 1961; Lee \& Pollack, 1978), require much more processing time than do young adults to achieve the same level of analytic performance (Ward \& Foley, 1984).

The results from the GEFT and classification tasks have both theoretical and practical implications. From a theoretical perspective, they indicate that the deleterious con- 
sequences of alcohol ingestion are most likely to be observed in time-limited tasks. A wide variety of deficits shown by intoxicated individuals may be due not so much to a disruption of the ability to perform cognitive/ perceptual operations as to a general slowing of the rate at which those operations can be performed. Further research should carefully control the time constraints of a variety of tasks in order to investigate this issue.

From a practical standpoint, it becomes important to know how aware individuals are that their rates of information processing have been slowed by alcohol. In the formal setting of a laboratory task, individuals appear to compensate by taking more time to perform the task. The extent to which individuals attempt to compensate in realworld settings is not clear, but this appears to be a topic worthy of study (see, e.g., Sher, 1985).

An additional practical implication of the present results is that alcohol appears to make individuals less resistant to the effects of distractors, such as loud music. When music was present, individuals who had consumed alcohol made fewer analytic responses overall and appeared to require more time to generate those responses. An important and neglected area of research concerns the interactive effects of alcohol with distractors commonly found in work and driving situations. Too much research has examined the effects of alcohol on performance of cognitive/perceptual tasks under formal laboratory conditions with few distractors. The effects on rate of information processing may be much more extreme under more realistic conditions.

The fact that music appears to increase analytic responding in sober adults and decrease analytic responding in those who have received alcohol is intriguing in that it supports findings from developmental studies. For example, Higgins and Turnure (1984) reported that the presence of extraneous music improved peformance in older children but lowered performance in young children. Participants who consumed alcohol in the present study were affected by the distraction of music in much the same way as were young children in Higgins and Turnure's study. Since it is likely that many individuals who are driving while under the influence of alcohol will be listening to music, the result has strong safety implications. Perhaps the number of alcohol-related accidents can be reduced by a reduction in the number of distractions present (e.g., music or conversation).

The only task that failed to reveal significant effects of alcohol was concept learning. There was a slight, though nonsignificant, slowing in the rate of concept acquisition. [Further tests also revealed that, in the alcohol group, subjects whose BAC was above the median needed more trial blocks to learn the concept than did those whose BAC was below the median; $F(1,12)=6.00, p<.05]$. However, alcohol did not shift adults to more holistic modes of learning. The discrepancy between the results for the classification and concept-learning tasks is probably not due to different BAC levels, since the levels were higher for the latter task whereas the deficit in performance was greater for the former. The failure to find a shift to more holistic responding in the concept-learning task is also unlikely to be due to a ceiling effect, since none of the groups approached the maximum of four analytic responses to the critical test faces.

The fact that alcohol affected classification performance but not concept-learning performance does not represent the first time that results with two such tasks have diverged. In previous studies, we have found that, as a group, learning-disabled children show more holistic patterns of classification than do their nondisabled peers of the same age (Ward, 1986b) but that, unlike younger nondisabled children (see Kemler Nelson, 1984, Experiment 4), they do not learn holistically structured problems more readily than analytic ones in concept-learning tasks (Ward, 1986a). In addition, adults who respond quickly show more holistic patterns in the classification task (J. D. Smith \& Kemler Nelson, 1984; Ward, 1983) but more analytic patterns in the concept-learning task (Ward \& Scott, in press). Thus, it may be that the classification and concept-learning tasks measure different aspects of holistic processing.

It is possible that classification and concept-learning tasks measure different levels of processing. On the surface, the classification task appears to involve more of a visual-analysis component concerned with breaking a stimulus into component dimensions. In contrast, the most salient aspect of the concept-learning task appears to be the more cognitive component of finding the rule that the experimenter has defined as correct for solving the problem. It is conceivable that alcohol, in the doses used in the present study, has a stronger effect on the former than on the latter. What these findings suggest is that, in addition to the practical value of studying the effects of alcohol, there may be a theoretical benefit in that alcohol may help to identify similarities and differences between tasks that are thought to measure similar processes.

\section{REFERENCES}

Axelrod, S., \& Cohen, L. D. (1961). Senescence and embedded-figure performance in vision and touch. Perceptual \& Motor Skills, 12, 283-288.

Burns, B., Shepp, B. E., McDonough, D., \& Wiener-Eyruch, W. K. (1978). The relation between stimulus analyzability and perceived dimensional structure. In G. H. Bower (Ed.), The psychology of learning and motivation (Vol. 12). New York: Academic Press.

FisHer, M. A. (1977). Dimensional interaction in hyperactive children: Classification of computer-displayed stimuli. Bulletin of the Psychonomic Society, 10, 433-446.

GARNER, W. R. (1970). The stimulus in information processing. American Psychologist, 25, 350-358.

GARNER, W. R. (1974). The processing of information and structure. Potomac, MD: Erlbaum.

Hamilton, P., Copeman, A. (1970). The effect of alcohol and noise on components of a tracking and monitoring task. British Journal of Psychology, 61, 149-156.

Harley, J. P., Cohen, R., \& Silverman, A. J. (1974). Field dependence and response to alcohol. Quarterly Journal of Studies on Alcohol, 35, 973-986.

Higgins, A. T., \&uRnure, J. E. (1984). Distractibility and concentration of attention in children's development. Child Development, $55,1799-1811$.

Kahneman, D., Ben-Ishai, R., Lotan, M. (1973). Relation of a 
test of attention to road accidents. Journal of Applied Psychology, 58, 113-115.

KEMLER, D. G. (1982). Classification in young and retarded children: The primacy of overall similarity relations. Child Development, 53, 768-779.

Kemler Nelson, D. G. (1984). The effect of intention on what concepts are acquired. Joumal of Verbal Leaming \& Verbal Behavior, 23, 734-759.

KogaN, N. (1973). Creativity and cognitive style: A lifespan perspective. In P. Baltes \& K. W. Schaie (Eds.), Life span developmental psychology: Personality and socialization. New York: Academic Press.

Kristofrerson, M. W. (1968). Effect of alcohol on perceptual field dependence. Journal of Abnormal Psychology, 73, 387-391.

LEE, J. A., \& PolLaCK, R. H. (1978). The effect of age on perceptual problem-solving strategies. Experimental Aging Research, 4, 37-54.

LOCKHEAD, G. R. (1966). Effects of dimensional redundancy on visual discrimination. Journal of Experimental Psychology, 72, 95-104.

MacArthur, R. D., \& SekULer, R. (1982). Alcohol and motion perception. Perception \& Psychophysics, 31, 502-505.

MeDIN, D. L., S SMTrH, E. E. (1981). Strategies and classification learning. Journal of Experimental Psychology: Learning, Memory, \& Cognition, 7, 241-253.

MiHAL, W. L., * BARRETT, G. V. (1976). Individual differences in perceptual information processing and their relation to automobile accident involvement. Journal of Applied Psychology, 61, 229-233.

MrTCHeLL, M. C. (1985). Alcohol-induced impairment of central nervous system function: Behavioral skills involved in driving. Journal of Studies on Alcohol (Suppl. No. 10), 109-116.

Mosxowrtz, H. (1973). Laboratory studies of the effects of alcohol on some variables related to driving. Journal of Safety Research, 5, 185-199.

Moskowitz, H., BuRNs, M. (1971). The effect of alcohol upon the psychological refractory period. Quarterly Journal of Studies on Alcohol, 32, 782-790.

Moskowitz, H., \& DePry, D. (1968). The effect of alcohol upon auditory vigilance and divided attention tasks. Quarterly Journal of Studies on Alcohol, 29, 54-63.

Moskowirz, H., MUrruy, J. T. (1976). Alcohol and backward masking of visual information. Joumal of Studies on Alcohol, 37, 40-45.

MoskowTIZ, H., \& SHARMA, S. (1974). Effects of alcohol on peripheral vision as a function of attention. Human Factors, 16, 174-180.

Oltman, P. K., RAskin, E., \& WitkIn, H. A. (1971). Group Embedded Figures Test. Palo Alto, CA: Consulting Psychologists Press.

SHEPARD, R. N. (1964). Attention and the metric structure of the stimulus space. Joumal of Mathematical Psychology, 1, 54-87.

SHEPP, B. E. (1978). From perceived similarity to dimensional structure: A new hypothesis about perceptual development. In E. Rosch \& B. Lloyd (Eds.), Cognition and categorization. Hillsdale, NJ: Erlbaum.

Shepp, B. E., BurNs, B., \& McDonough, D. (1980). The relation of stimulus structure to perceptual and cognitive development: Further tests of a separability hypothesis. In F. Wilkening, J. Becker, \& T. Trabasso (Eds.), Information integration by children. Hillsdale, NJ: Erlbaum.

SHER, K. J. (1985). Subjective effects of alcohol: The influence of setting and individual differences in alcohol expectancies. Joumal of Studies on Alcohol, 46, 137-146.
Shunar, D. (1978). Psychology on the road: The human factor in traffic safety. New York: Wiley.

SmTH, J. D., Kemler Nelson, D. G. (1984). Overall similarity in adults' classification: The child in all of us. Joumal of Experimental Psychology: General, 113, 137-159.

SmitH, L. B., KeMLER, D. G. (1977). Developmental trends in free classification: Evidence for a new conceptualization of perceptual development. Journal of Experimental Child Psychology, 24, 279-298.

U.S. DEPARTMENT OF TrANSPORTATION (1985). Alcohol and highway safety 1984: A review of the state of the knowledge (DOT-HS-806569). Washington DC: U.S. Government Printing Office.

WARD, T. B. (1978). Dimensional responding in children and adults as a function of stimulus and response variables. (Unpublished doctoral dissertation, University of Wisconsin-Madison). Dissertation Abstracts International, 3, 4565B. (University Microfilms No. 78-23, 091)

WARD, T. B. (1980). Separable and integral responding by children and adults to the dimensions of length and density. Child Development, $51,676-684$.

WARD, T. B. (1983). Response tempo and separable-integral responding: Evidence for an integral-to-separable processing sequence in visual perception. Journal of Experimental Psychology: Human Perception \& Performance, 9, 103-112.

Ward, T. B. (1985). Individual differences in processing stimulus dimensions: Relation to selective processing abilities. Perception \& Psychophysics, 37, 471-482.

WARD, T. B. (1986a, March). Acquisition of criterial attribute and family-resemblance concepts by learning disabled and nondisabled children. Paper presented at the meeting of the Southwest Society for Research in Human Development, San Antonio, Texas.

WARD, T. B. (1986b). Classifying by learning disabled and nondisabled children: Use of overall similarity versus dimensional relations. Bulletin of the Psychonomic Society, 24, 131-134.

WARD, T. B., FoLEY, C. M. (1984, March). Analytic and holistic perception in younger and older adults. Paper presented at the meeting of the Southwest Society for Research in Human Development, Denver, Colorado.

WARD, T. B., Folry, C. M., \& COLE, J. (1986). Classifying multidimensional stimuli: Stimulus, task and observer factors. Joumal of Experimental Psychology: Human Perception \& Performance, 12, 211-225.

WARD, T. B., ScotT, J. (in press). Analytic and holistic modes of learning family-resemblance concepts. Memory \& Cognition.

\section{NOTE}

1. Tighter experimental control might have been achieved by using exactly the same music for all participants. However, the purpose here was to use music that the participant would like and therefore would be more likely to become involved with and distracted by during the performance of the task.

(Manuscript received March 31, 1986; revision accepted for publication November 4, 1986.) 